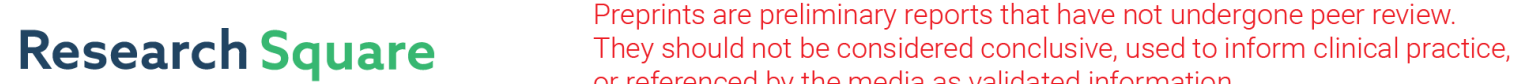 \\ or referenced by the media as validated information. \\ Physical Layer Security for Cooperative NOMA Networks via Nakagami-m Fading Channels
}

Wided Hadj Alouane ( $\square$ wided.hadjalouane@gmail.com )

SUP'COM, COSIM LAB https://orcid.org/0000-0001-8066-1623

\section{Research Article}

Keywords: non-orthogonal multiple access (NOMA), Nakagami-m fading channel, amplify-and-forward, Decode-and-forward, strictly positive secrecy capacity

Posted Date: April 15th, 2021

DOI: https://doi.org/10.21203/rs.3.rs-247227/v1

License: (c) (i) This work is licensed under a Creative Commons Attribution 4.0 International License. Read Full License 


\title{
Physical Layer Security for Cooperative NOMA Networks via Nakagami-m Fading Channels
}

\author{
Wided Hadj Alouane
}

Received: date / Accepted: date

\begin{abstract}
In this paper, we investigate the physical layer security in cooperative non-orthogonal multiple access (NOMA) networks over Nakagami$\mathrm{m}$ fading channels. Both amplify-and-forward (AF) and decode-and-forward (DF) protocols are studied. More particularly, closed-form exact and asymptotic expressions for strictly positive secrecy capacity are provided considering NOMA-AF and NOMA-DF relaying systems. Numerical results are presented to justify the accuracy of the obtained theoretical analysis. These results show that NOMA-AF and NOMA-DF relaying networks have a similar secrecy performance.
\end{abstract}

Keywords non-orthogonal multiple access (NOMA) · Nakagami-m fading channel $\cdot$ amplify-and-forward $\cdot$ Decode-and-forward $\cdot$ strictly positive secrecy capacity

\section{Introduction}

Non-orthogonal multiple access (NOMA) has recently been envisaged as a promising solution to greatly enhance the spectral efficiency and ensure massive connectivity of fifth generation (5G) mobile communications [1-3]. Unlike the conventional orthogonal multiple access (OMA), NOMA invites multiple users to share the same resources such as time, frequency and code,but it uses multiple access (MA) in power domain [4]. NOMA technique aims to attribute different power levels to different users based on their channels' conditions. More specifically, a less power is allocated to users with good channel quality. These users apply successive interference cancellation (SIC) operation to detect their own messages and to subtract the multi-user interference.

W. Hadj Alouane

University of Carthage, COSIM Laboratory, Higher School of Communication of Tunis

Ariana, Tunisia

E-mail: wided.hadjalouane@gmail.com 
On the other hand, a more power is allocated to users with poor channel conditions to detect their own data by considering the information of the other users as noise [5]. To improve the performance of NOMA networks, cooperative transmission was introduced to NOMA in [6-8]. In [9], a hybrid decode-and-forward (DF) and AF protocol was analyzed in multi-relay NOMA system over Rayleigh fading channels. In [10], the performance of NOMA-AF relaying networks was studied in terms of outage probability considering independent Rayleigh fading links. The authors of [11] investigated the effect of two-stage relay selection method on the performance of NOMA-DF relaying system via Rayleigh fading channels. More particularly, a NOMA-DF system with two-stage relay selection significantly outperforms a NOMA-DF system with max-min relay selection. Recently, huge efforts have been devoted to the security issues in wireless communication networks, due to the open nature of wireless channels which makes wireless transmissions vulnerable to eavesdropping. Physical layer security has emerged as an indispensable technology to improve secrecy over wireless channels [12]. In this context, physical layer security was widely employed in previous works using different techniques such as cooperative diversity [13], energy harvesting [14], multiple input multiple output (MIMO) and radio cognitive [15]. However, NOMA technology was not considered in these previous contributions. Lately, the authors of [16] have investigated the physical layer security in fifth generation (5G) NOMA networks. Moreover, the authors of [17] have provided a new design of NOMA under secrecy considerations. They also derived transmission rates, optimal designs of decoding order and power allocated to each user node considering Rayleigh fading channels.

The performance of secure NOMA-AF system and secure NOMA-DF system through Rayleigh fading channels was evaluated in [18] in terms of secrecy outage probability and strictly positive secrecy capacity.

All the above-mentioned contributions studied the secrecy performance of NOMA system considering

Rayleigh fading channels. However, it is worth mentioning that to the best of the authors' knowledge there is no published work concerning the performance analysis of secure NOMA-AF and NOMA-DF relaying systems over Nakagami-m fading channels. In this paper, we analyze the secrecy performance of cooperative NOMA relaying networks where AF and DF protocols are considered under Nakagami-m fading channels with integer $\mathrm{m}$ values. The main contributions of this paper are detailed as follows.

- We present the performance analysis of secure NOMA relaying communications networks considering both $\mathrm{AF}$ and $\mathrm{DF}$ relaying protocols over Nakagami-m fading links with integer $m$ factor. The Nakagami-m fading is well employed in practical wireless applications. It can present different fading models such as Rayleigh fading. We further determine the impact of Nakagami-m factor on the secrecy performance. 
- We evaluate NOMA-AF and NOMA-DF systems consisting on one base station, a single relay, two users and one eavesdropper. We consider that the wireless data transmission is intercepted by an eavesdropper.

- We provide the secrecy performance of NOMA-AF and NOMA-DF relaying networks over Nakagami-m fading channels in terms of closed-form exact and asymptotic expressions for strictly positive secrecy capacity.

- The numerical results show that the secrecy performance of both relaying systems: NOMA-AF and NOMA-DF boosts as the Nakagami-m fading parameter rises. However, Nakagami-m fading factor does not have a considerable effect on secrecy performance when enhancing the signal-to-noise ratio (SNR) received at eavesdropper.

- Results also show that a comparable secrecy performance is obtained for NOMA-AF and NOMA-DF relaying networks especially for high SNR values.

The rest of the paper is arranged as follows. In section 2, we depict the system model. Exact and asymptotic expressions for strictly positive secrecy capacity of NOMA-AF and NOMA-DF relaying systems are derived in section 3 and section 4 , respectively. Section 5 introduces various numerical results. Eventually, the paper concludes with section 6 .

\section{System Model}

In this section, we present a secure NOMA system operating over Nakagami-m fading channels using both protocols AF and DF. We consider the same system presented in [18]. Fig. 1 shows that the system contains one base station $(B S)$, one relay $(R)$, a strong user $\left(U_{1}\right)$, a poor user $\left(U_{2}\right)$ and one eavesdropper $(E)$. We consider a cellular network where the base station is placed in the center of the cell and terminal receiver nodes (strong user, poor user and eavesdropper) are very near to the cell bound. We assume that there are not direct connections between the base station and both users and eavesdropper. Moreover, we consider that all nodes of the network are equipped with a single antenna an all channels suffer from Nakagami-m fading. The probability density function $(\mathrm{PDF})$ and cumulative distribution function (CDF) for Nakagami-m fading channels are respectively written as

$$
f_{x}(X)=\left(\frac{m}{\bar{X}}\right)^{m} \frac{X^{m-1}}{\Gamma(m)} \exp \left(\frac{-m X}{\bar{X}}\right) ; X \geq 0
$$

and

$$
F_{x}(X)=\Gamma\left(m, \frac{m X}{\bar{X}}\right)=1-\sum_{z=0}^{m-1} \frac{1}{z !} \exp \left(\frac{-m X}{\bar{X}}\right)\left(\frac{m X}{\bar{X}}\right)^{z}
$$

where $m$ is the Nakagami- $m$ fading parameter and $\Gamma(.,$.$) is the complete$ Gamma function given by $\Gamma(\theta)=\int_{0}^{\infty} y^{\theta-1} \exp (-y) d y$.

In the first time-interval, the base station broadcasts a combined signal $\epsilon_{1} x_{1}+$ $\epsilon_{2} x_{2}$ to the relay where $x_{i}(i=1,2)$ is the unit power signal received at user 


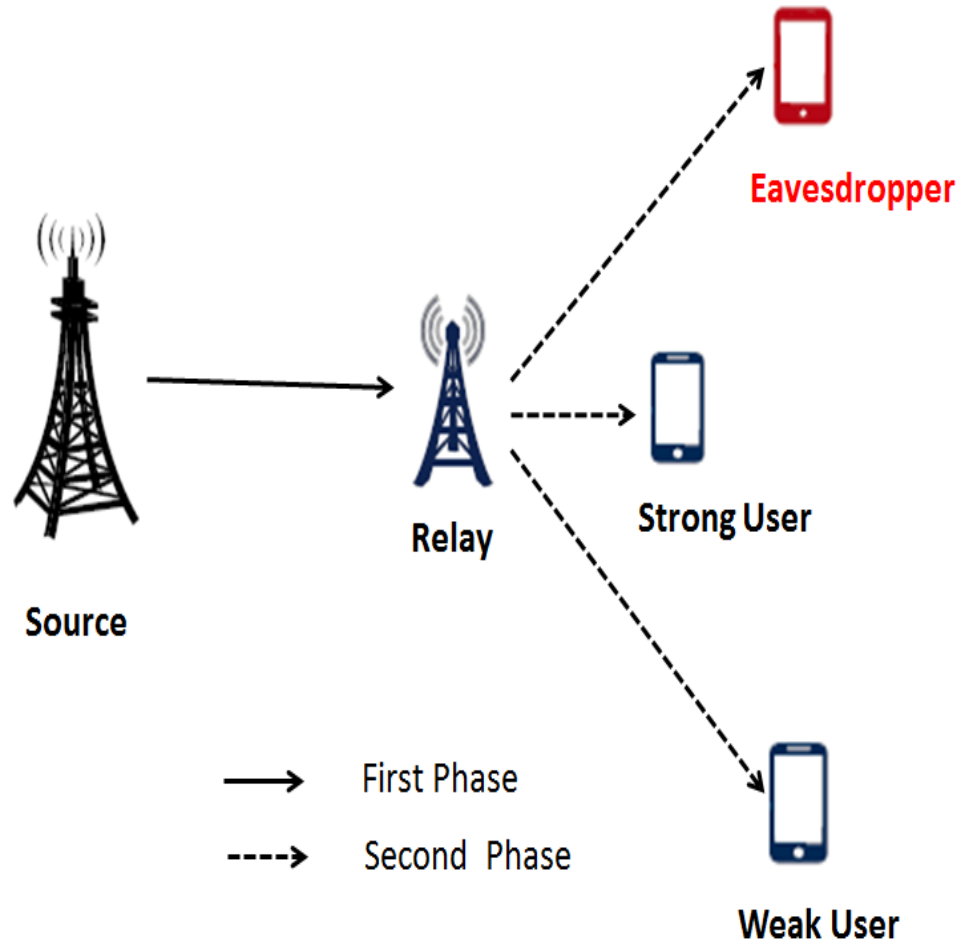

Fig. 1: System architecture

$i$ and $\epsilon_{i}(i=1,2)$ is the power allocation factor. To satisfy $U_{1}$ 's QoS requirements, we consider that $\epsilon_{1} \geq \epsilon_{2}$ and define the power allocation factors as $\epsilon_{1}^{2}+\epsilon_{2}^{2}=1$ [19]. Therefore, the relay signal can be written as

$$
z_{R}=\epsilon_{1} x_{1} h_{R} \sqrt{P_{S}}+\epsilon_{2} x_{2} h_{R} \sqrt{P_{S}}+n_{R}
$$

where $h_{R}$ stands for the channel coefficient between the base station and the relay and $n_{R}$ denotes the Additive Gaussian Noise (AWGN) with zero mean and variance $N_{0}$. In the following, we present the transmission schemes considering respectively $\mathrm{AF}$ and $\mathrm{DF}$ protocols.

\subsection{Amplify-and-Forward}

In the second time-interval, the relay transmits the amplified signal to both users. Hence, the received signal at the user $i$ can be expressed as

$$
z_{R, i}^{A F}=G z_{R} h_{R, i}+n_{R, i}
$$

where $G$ is the amplifying gain denoted as $G=\sqrt{\frac{P_{R}}{P_{S}\left|h_{R}\right|^{2}+N_{0}}}[20], h_{R, i}$ stands for the channel coefficient between the relay and user $U_{i}, n_{R, i}$ is the AWGN 
with zero mean and variance $N_{0}$ and $P_{R}$ is the transmit relay power. We consider that $P_{R}=P_{S}$, hence $G=\sqrt{\frac{1}{\left|h_{R}\right|^{2}+\frac{1}{\mu}}}$ where $\mu=\frac{P_{S}}{N_{0}}=\frac{P_{R}}{N_{0}}$ is the average SNR of legitimate channels. $h_{R, i}$ is the small-scale fading gain of the link between the relay and user $i$. Owing to the broadcast nature of wireless communications, the eavesdropper can also receive the relay signal. Then, the signal received at $E$ can be given as

$$
z_{R, E}^{A F}=G z_{R} h_{R, E}+n_{R E}
$$

Where $h_{R, E}$ represents the channel coefficient between the relay and eavesdropper and $n_{R, E}$ denotes an AWGN with variance $N_{E}$. According to $[21,22]$, the user $i$ applies a successive interference cancellation (SIC) to decode the signal $x_{i}$. Then, the channel capacity from the cooperating node to user $i$ considering the AF protocol can be written as

$$
C a_{R, i}^{A F}=\frac{1}{2} \log _{2}\left(1+\Gamma_{R, i}^{A F}\right)
$$

where $\Gamma_{R, 1}^{A F}=\frac{\left|h_{R, 1}\right|^{2}\left|h_{R}\right|^{2} \epsilon_{1}^{2}}{\left|h_{R, 1}\right|^{2}\left|h_{R}\right|^{2} \epsilon_{2}^{2}+\frac{1}{\mu}\left|h_{R, 1}\right|^{2}+\frac{1}{\mu}\left|h_{R}\right|^{2}+\frac{1}{\mu^{2}}}$ is the instantaneous signal-tointerference-pulse-noise ratio (SINR) at $U_{1}$ and $\Gamma_{R, 2}^{A F}=\frac{\mu\left|h_{R, 2}\right|^{2}\left|h_{R}\right|^{2} \epsilon_{2}^{2}}{\left|h_{R, 2}\right|^{2}+\left|h_{R}\right|^{2}+\frac{1}{\mu}}$ denotes the received SNR at user $U_{2}$.

Similar to $[16,22]$, we assume the worst case and consider that eavesdropper $E$ has the multiuser detection ability. Indeed, the eavesdropper uses a parallel interference cancellation (PIC) technique in order to differentiate the superimposed mixture. Hence, the transmission capacity from the relay to eavesdropper can be written as

$$
C a_{E, i}^{A F}=\frac{1}{2} \log _{2}\left(1+\Gamma_{E, i}^{A F}\right)
$$

where $\Gamma_{E, i}^{A F}=\frac{\mu \mu_{E}\left|h_{R, E}\right|^{2}\left|h_{R}\right|^{2} \epsilon_{i}^{2}}{\mu_{E}\left|h_{R, E}\right|^{2}+\mu\left|h_{R}\right|^{2}+1}$ denotes the received SNR at eavesdropper and $\mu_{E}=\frac{P_{R}}{N_{E}}$ represents the average SNR of the eavesdropper link. According to (6) and (7), the secrecy rate of the NOMA-AF systems for user i can be written as

$$
C a_{i}^{A F}=\left[C a_{R, i}^{A F}-C a_{E, i}^{A F}\right]^{+}
$$

where $[x]^{+}=\max (x, 0)$.

\subsection{Decode-and-Forward}

The relay decodes the received signal and then transmits the re-encoded signal to users and eavesdropper. Hence, the signals received at $U_{i}$ and $E$ can be written as 


$$
z_{R, \beta}^{D F}=h_{R, \beta}\left(\epsilon_{1} x_{1}+\epsilon_{2} x_{2}\right) \sqrt{P_{S}}+n_{R, \beta}
$$

where $\beta \in\{i, E\}$.

The transmission capacity of secure NOMA-DF relaying systems is $\min \left\{C a_{B S, R}, C a_{R, U_{i}}\right\}$, where $C a_{B S, R}$ and $C a_{R, U_{i}}$ set the channel capacity from base station to relay and relay to user $U_{i}$, respectively. Then, the capacity of the main links can be expressed as

$$
C a_{B S, U i}^{D F, i}=\frac{1}{2} \log _{2}\left(1+\min \left\{\frac{\mu\left|h_{R}\right|^{2} \epsilon_{1}^{2}}{\mu\left|h_{R}\right|^{2} \epsilon_{2}^{2}+1}, \frac{\mu\left|h_{R, 1}\right|^{2} \epsilon_{1}^{2}}{\mu\left|h_{R, 1}\right|^{2} \epsilon_{2}^{2}+1,}\right\}\right)
$$

and

$$
C a_{B S, U i}^{D F, i}=\frac{1}{2} \log _{2}\left(1+\min \left\{\mu\left|h_{R}\right|^{2} \epsilon_{2}^{2}, \mu\left|h_{R, 2}\right|^{2} \epsilon_{2}^{2}\right\}\right)
$$

In the meantime, the capacity of the eavesdropper channel can be written as

$$
C a_{R, E}^{D F, i}=\frac{1}{2} \log _{2}\left(1+\mu_{E}\left|h_{R, E}\right|^{2} \epsilon_{i}^{2}\right)
$$

Then, the overall secrecy capacity of NOMA-DF relaying system can be given as

$$
C a_{i}^{D F}=\left[C a_{B S, U i}^{D F, i}-C a_{R, E}^{D F, i}\right]^{+}
$$

\section{Strictly Positive Secrecy Capacity}

In this section, we investigate the secrecy performance of both systems: NOMAAF and NOMA-DF by providing an analytical expression of strictly positive secrecy capacity $(S P S C a)$.

\subsection{Amplify-and-Forward}

The strictly positive secrecy capacity for NOMA-AF relaying system can be expressed as

$$
S P S C a^{A F}=\mathcal{P}\left(C a_{1}^{A F}>0, C a_{2}^{A F}>0\right)
$$

$C a_{1}^{A F}$ and $C a_{2}^{A F}$ are respectively given as

$$
C a_{1}^{A F}=\frac{1+\Gamma_{R, 1}^{A F}}{1+\Gamma_{E, 1}^{A F}}
$$

and

$$
C a_{2}^{A F}=\frac{1+\Gamma_{R, 2}^{A F}}{1+\Gamma_{E, 2}^{A F}}
$$

By using (15) and (16), SPSCa ${ }^{A F}$ can be re-written as

$$
S P S C a^{A F}=\mathcal{P}\left(\Gamma_{R, 1}^{A F}>\Gamma_{E, 1}^{A F}, \Gamma_{R, 2}^{A F}>\Gamma_{E, 2}^{A F}\right)
$$


For high SNR regime, $\Gamma_{R, 1}^{A F}, \Gamma_{R, 2}^{A F}$ and $\Gamma_{E, i}^{A F}(i=1,2)$ can be approximated as

$$
\begin{gathered}
\Gamma_{R, 1}^{A F}<\frac{\epsilon_{1}^{2}}{\epsilon_{2}^{2}} \\
\Gamma_{R, 2}^{A F}<\frac{\mu \mu\left|h_{R}\right|^{2}\left|h_{R, 2}\right|^{2} \epsilon_{2}^{2}}{\mu\left|h_{R}\right|^{2}+\mu\left|h_{R, 2}\right|^{2}}
\end{gathered}
$$

and

$$
\Gamma_{E, i}^{A F}<\frac{\mu \mu_{E}\left|h_{R}\right|^{2}\left|h_{R, E}\right|^{2} \epsilon_{i}^{2}}{\mu\left|h_{R}\right|^{2}+\mu_{E}\left|h_{R, E}\right|^{2}}
$$

respectively. By applying the inequality $x y /(x+y) \leq \min \{x, y\},(19)$ and $(20)$ can be expressed as

$$
\Gamma_{R, 2}^{A F}<\epsilon_{2}^{2} \min \left\{\mu\left|h_{R}\right|^{2}, \mu\left|h_{R, 2}\right|^{2}\right\}
$$

and

$$
\Gamma_{E, i}^{A F}<\epsilon_{i}^{2} \min \left\{\mu\left|h_{R}\right|^{2}, \mu_{E}\left|h_{R, E}\right|^{2}\right\}
$$

respectively.

Hence, $S P S C a^{A F}$ can be given as

$$
\begin{aligned}
S P S C a^{A F} & \approx \mathcal{P}\left(\frac{1}{\epsilon_{2}^{2}}>\min \left\{\mu\left|h_{R}\right|^{2}, \mu_{E}\left|h_{R, E}\right|^{2}\right\}, \min \left\{\mu\left|h_{R}\right|^{2}, \mu\left|h_{R, 2}\right|^{2}\right\}\right. \\
& \left.>\min \left\{\mu\left|h_{R}\right|^{2}, \mu_{E}\left|h_{R, E}\right|^{2}\right\}\right)
\end{aligned}
$$

It is difficult to determine a closed-form expression for the strictly positive secrecy capacity. Hence, we use the equality $\mathcal{P}\left(\phi_{1}, \phi_{2}\right)=\mathcal{P}\left(\phi_{1}\right)-\mathcal{P}\left(\phi_{1}, \bar{\phi}_{2}\right)$ in order to simplify the mathematical analysis, such that $\bar{\phi}_{2}$ represents the complementary event of $\phi_{2}, \phi_{1}=\min \left\{\mu\left|h_{R}\right|^{2}, \mu\left|h_{R, 2}\right|^{2}\right\}>\min \left\{\mu\left|h_{R}\right|^{2}, \mu_{E}\left|h_{R, E}\right|^{2}\right\}$ and $\phi_{2}=\min \left\{\mu\left|h_{R}\right|^{2}, \mu_{E}\left|h_{R, E}\right|^{2}\right\}<\frac{1}{\epsilon_{2}^{2}}$.

Therefore, $S P S C a^{A F}$ can be written as

$$
\begin{aligned}
S P S C a^{A F} & =\mathcal{P}\left(\min \left\{\mu\left|h_{R}\right|^{2}, \mu\left|h_{R, 2}\right|^{2}\right\}>\min \left\{\mu\left|h_{R}\right|^{2}, \mu_{E}\left|h_{R, E}\right|^{2}\right\}\right) \\
& -\mathcal{P}\left(\min \left\{\mu\left|h_{R}\right|^{2}, \mu\left|h_{R, 2}\right|^{2}\right\}>\min \left\{\mu\left|h_{R}\right|^{2},\right.\right. \\
& \left.\left.\mu_{E}\left|h_{R, E}\right|^{2}\right\}, \min \left\{\mu\left|h_{R}\right|^{2}, \mu_{E}\left|h_{R, E}\right|^{2}\right\}>\frac{1}{\epsilon_{2}^{2}}\right)
\end{aligned}
$$

We denote $J_{1}=\mathcal{P}\left(\min \left\{\mu\left|h_{R}\right|^{2}, \mu\left|h_{R, 2}\right|^{2}\right\}>\min \left\{\mu\left|h_{R}\right|^{2}, \mu_{E}\left|h_{R, E}\right|^{2}\right\}\right)$ and $J_{2}=\mathcal{P}\left(\min \left\{\mu\left|h_{R}\right|^{2}, \mu\left|h_{R, 2}\right|^{2}\right\}>\min \left\{\mu\left|h_{R}\right|^{2}, \mu_{E}\left|h_{R, E}\right|^{2}\right\}, \min \left\{\mu\left|h_{R}\right|^{2}\right.\right.$, $\left.\left.\mu_{E}\left|h_{R, E}\right|^{2}\right\}>\frac{1}{\epsilon_{2}^{2}}\right)$.

$J_{1}$ can be re-expressed as

$$
\begin{aligned}
J_{1} & =\mathcal{P}\left(\min \left\{\mu\left|h_{R}\right|^{2}, \mu\left|h_{R, 2}\right|^{2}\right\}>\mu_{E}\left|h_{R, E}\right|^{2}\right) \\
& =\mathcal{P}\left(\left|h_{R}\right|^{2}>\frac{\mu_{E}}{\mu}\left|h_{R, E}\right|^{2},\left|h_{R, 2}\right|^{2}>\frac{\mu_{E}}{\mu}\left|h_{R, E}\right|^{2}\right)
\end{aligned}
$$


By using the PDF and CDF for the Nakagami-m fading channels, the above equation can be written as

$$
\begin{aligned}
J_{1} & =\int_{0}^{\infty} \sum_{k=0}^{m-1} \exp \left(-m x \mu_{E}\left(\frac{\alpha_{0}+\alpha_{2}}{\mu}\right)\right) x^{k} \\
& \left(m \mu_{E}\left(\frac{\alpha_{0}+\alpha_{2}}{\mu}\right)\right)^{k}\left(m \alpha_{E}\right)^{m} \frac{x^{m-1}}{\Gamma(m) k !} \exp \left(-m x \alpha_{E}\right) d x \\
& =\sum_{k=0}^{m-1} \frac{B^{m}}{\Gamma(m) k !} A^{k} \int_{0}^{\infty} \exp (-A x) \exp (-B x) x^{m+k-1} d x
\end{aligned}
$$

where $\alpha_{0}, \alpha_{2}$ and $\alpha_{E}$ are respectively the Nakagami-m channel parameters corresponding to $\left|h_{R}\right|^{2},\left|h_{R, 2}\right|^{2}$ and $\left|h_{R, E}\right|^{2}, A=\frac{m\left(\alpha_{0}+\alpha_{2}\right) \mu_{E}}{\mu}$ and $B=m \alpha_{E}$. The integral presented in the above equation can be resolved as $\int_{0}^{\infty} \exp (-A x) \exp (-B x) x^{m+k-1} d x=(A+B)^{-m-k} \Gamma(m+k)$ where $\Gamma($. represents the Gamma function. Hence, $J_{1}$ can be written as

$$
J_{1}=\sum_{k=0}^{m-1} \frac{B^{m}}{\Gamma(m) k !} A^{k}(A+B)^{-m-k} \Gamma(m+k)
$$

$J_{2}$ can be written as

$$
\begin{aligned}
J_{2} & =\mathcal{P}\left(\min \left\{\mu\left|h_{R}\right|^{2}, \mu\left|h_{R, 2}\right|^{2}\right\}>\mu_{E}\left|h_{R, E}\right|^{2}, \mu_{E}\left|h_{R, E}\right|^{2}>\frac{1}{\epsilon_{2}^{2}}\right) \\
& =\mathcal{P}\left(\left|h_{R}\right|^{2}>\frac{\mu_{E}}{\mu}\left|h_{R, E}\right|^{2},\left|h_{R, 2}\right|^{2}>\frac{\mu_{E}}{\mu}\left|h_{R, E}\right|^{2},\left|h_{R, E}\right|^{2}>\frac{1}{\mu_{E} \epsilon_{2}^{2}}\right)
\end{aligned}
$$

$J_{2}$ can be expressed for Nakagami-m fading channels as

$$
J_{2}=\sum_{k=0}^{m-1} \frac{B^{m}}{\Gamma(m) k !} A^{k} \int_{C}^{\infty} \exp (-A x) \exp (-B x) x^{m+k-1} d x
$$

where $C=\frac{1}{\mu_{E} \epsilon_{2}^{2}}$ and $\int_{C}^{\infty} \exp (-A x) \exp (-B x) x^{m+k-1} d x=(A+B)^{-m-k}$ $\Gamma(m+k,(A+B) C)$.

Therefore, $J_{2}$ can be obtained as

$$
J_{2}=\sum_{k=0}^{m-1} \frac{B^{m}}{\Gamma(m) k !} A^{k}(A+B)^{-m-k} \Gamma(m+k,(A+B) C)
$$

By using the equations (27) and (30), the strictly positive secrecy capacity for NOMA-AF relaying network over Nakagami-m fading channels can be given as 


$$
\begin{aligned}
S P S C a^{A F} & =J_{1}-J_{2} \\
& =\sum_{k=0}^{m-1} \frac{B^{m}}{\Gamma(m) k !} A^{k}(A+B)^{-m-k} \\
& (\Gamma(m+k)-\Gamma(m+k,(A+B) C))
\end{aligned}
$$

\subsection{Decode-and-Forward}

The strictly positive secrecy capacity for NOMA-DF relaying system over Nakagami-m fading channels can be expressed as

$$
\begin{aligned}
S P S C a^{D F} & =\mathcal{P}\left(\mu_{E}\left|h_{R, E}\right|^{2} \epsilon_{2}^{2}<1, \min \left\{\left|h_{R}\right|^{2},\left|h_{R, 2}\right|^{2}\right\}>\frac{\mu_{E}}{\mu}\left|h_{R, E}\right|^{2}\right) \\
& =\mathcal{P}\left(\left|h_{R, E}\right|^{2}<\frac{1}{\mu_{E} \epsilon_{2}^{2}},\left|h_{R}\right|^{2}>\frac{\mu_{E}}{\mu}\left|h_{R, E}\right|^{2},\left|h_{R, 2}\right|^{2}>\frac{\mu_{E}}{\mu}\left|h_{R, E}\right|^{2}\right)
\end{aligned}
$$

$S P S C a^{D F}$ can be written as

$$
S P S C a^{D F}=\sum_{k=0}^{m-1} \frac{B^{m}}{\Gamma(m) k !} A^{k} \int_{0}^{C} \exp (-A x) \exp (-B x) x^{m+k-1} d x
$$

where the expressions of $A$ and $B$ are presented after (26), and the expression of $C$ is presented after(29). The above integral can be calculated as

$$
\begin{aligned}
& \int_{0}^{C} \exp (-A x) \exp (-B x) x^{m+k-1} d x \\
& =(A+B)^{-m-k}(\Gamma(m+k)-\Gamma(m+k,(A+B) C))
\end{aligned}
$$

Hence, the strictly positive secrecy capacity for NOMA-DF relaying network over Nakagami-m fading channels can be given as

$S P S C a^{D F}=\sum_{k=0}^{m-1} \frac{B^{m}}{\Gamma(m) k !} A^{k}(A+B)^{-m-k}(\Gamma(m+k)-\Gamma(m+k,(A+B) C))$

\section{Asymptotic Strictly Positive Secrecy Capacity}

In this section, we provide asymptotic expressions for strictly positive secrecy capacity considering NOMA-AF and NOMA-DF relaying schemes. 
4.1 Amplify-and-Forward

When $\mu \rightarrow \infty$, the equation (26) can be written as

$$
J_{1} \approx \frac{B^{m}}{\Gamma(m)} \int_{0}^{\infty} \exp (-B x) x^{m-1} d x
$$

The above integral can be solved as $\int_{0}^{\infty} \exp (-B x) x^{m-1} d x=B^{-m} \Gamma(m)$. Hence, $J_{1}=1$. On the other hand, the equation (29) can be given as

$$
J_{2} \approx \frac{B^{m}}{\Gamma(m)} \int_{C}^{\infty} \exp (-B x) x^{m-1} d x=\frac{\Gamma(m, B C)}{\Gamma(m)}
$$

where $\int_{C}^{\infty} \exp (-B x) x^{m-1} d x=B^{-m} \Gamma(m, B C)$. Therefore, the strictly positive secrecy capacity for NOMA-AF relaying system can be asymptotically expressed as

$$
S P S C a^{A F}=J_{1}-J_{2}=1-\frac{\Gamma(m, B C)}{\Gamma(m)}
$$

\subsection{Decode-and-Forward}

Similarly, the asymptotic strictly positive secrecy capacity for NOMA-DF relaying system over Nakagami-m fading channels can be approximated as

$$
S P S C a^{D F} \approx \frac{B^{m}}{\Gamma(m)} \int_{0}^{C} \exp (-B x) x^{m-1} d x
$$

The above integral can be calculated as

$\int_{0}^{C} \exp (-B x) x^{m-1} d x=B^{-m}(\Gamma(m)-\Gamma(m, B C))$.

Thus, the asymptotic $S P S C a^{D F}$ can be written as

$$
S P S C a^{D F}=1-\frac{\Gamma(m, B C)}{\Gamma(m)}
$$

\section{Numerical Results}

In this section, we present some numerical results to reveal the secrecy performance for both systems: NOMA-AF and NOMA-DF over Nakagami-m fading channels.

Fig. 2 presents the strictly positive secrecy capacity for NOMA-AF relaying system when varying the Nakagami-m fading parameter $(m=1,2,3,4)$ and fixing $\epsilon_{1}=0.85, \mu_{E}=0 d B$ and $\alpha_{0}=\alpha_{2}=\alpha_{E}=1$. From this figure, we can observe that the greater the value of Nakagami-m parameter $m$, the higher the strictly positive secrecy capacity. Hence, the variation of Nakagami$\mathrm{m}$ parameter $m$ can have a significant effect on the secrecy performance. 


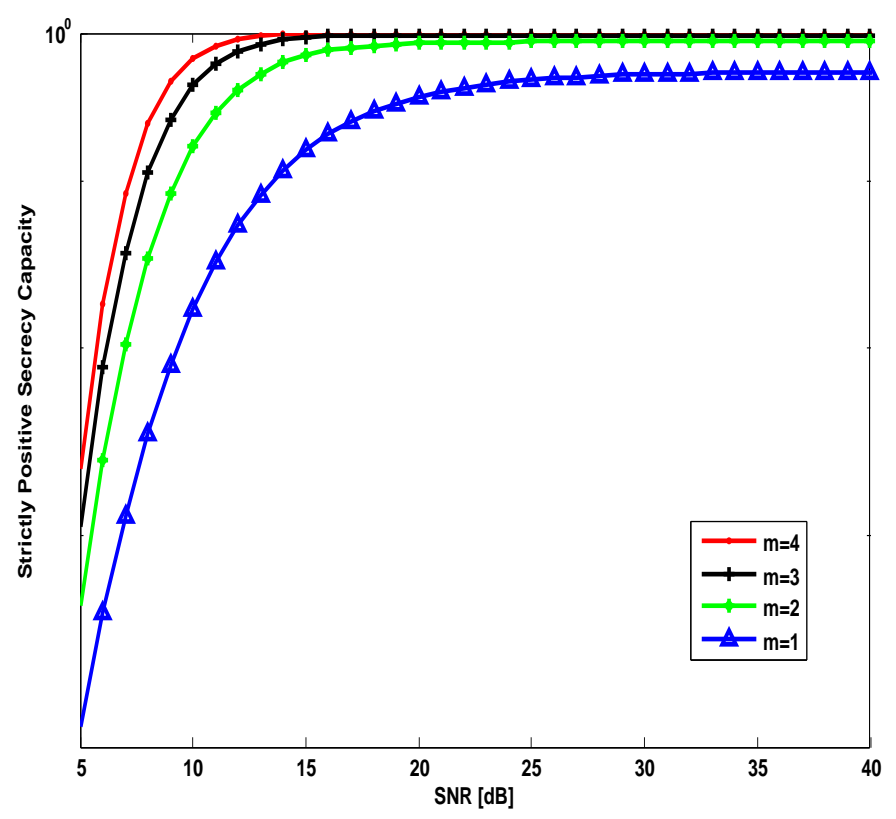

Fig. 2: Strictly Positive Secrecy Capacity for NOMA-AF relaying system when varying the Nakagami-m fading parameter $m$ and fixing $\epsilon_{1}=0.85, \mu_{E}=0 d B$ and $\alpha_{0}=\alpha_{2}=\alpha_{E}=1$

In Fig.3, we plot the strictly positive secrecy capacity curves for NOMAAF relaying system for different values of $\epsilon_{1}$ and $\mu_{E}$ assuming that $m=2$ and $\alpha_{0}=\alpha_{2}=\alpha_{E}=1$.

We can notice from this figure that the secrecy performance improves as increasing $\epsilon_{1}$ and worsens as rising $\mu_{E}$. Fig.3 also shows that $\epsilon_{1}$ does not affect the strictly positive secrecy capacity when increasing $\mu_{E}$.

Fig.4 illustrates the strictly positive secrecy capacity when varying $\mu_{E}$ for different values of Nakagami-m fading parameter $(m=1,2)$ considering that $\epsilon_{1}=0.95$ and $\alpha_{0}=\alpha_{2}=\alpha_{E}=1$. As expected, the strictly positive secrecy capacity enhances as $\mu_{E}$ decreases.

A comparison between NOMA-AF relaying system and NOMA-DF relaying system is presented in Fig.5 considering Nakagami-m fading channels.

In this figure, we consider multiple values of Nakagami-m parameter $(m=1,2,3), \mu_{E}=0 d B, \epsilon_{1}=0.85$ and $\alpha_{0}=\alpha_{2}=\alpha_{E}=1$. This figure shows that both systems: NOMA-AF and NOMA-DF have a similar secrecy performance especially for high values of SNR. As illustrated in asymptotic 


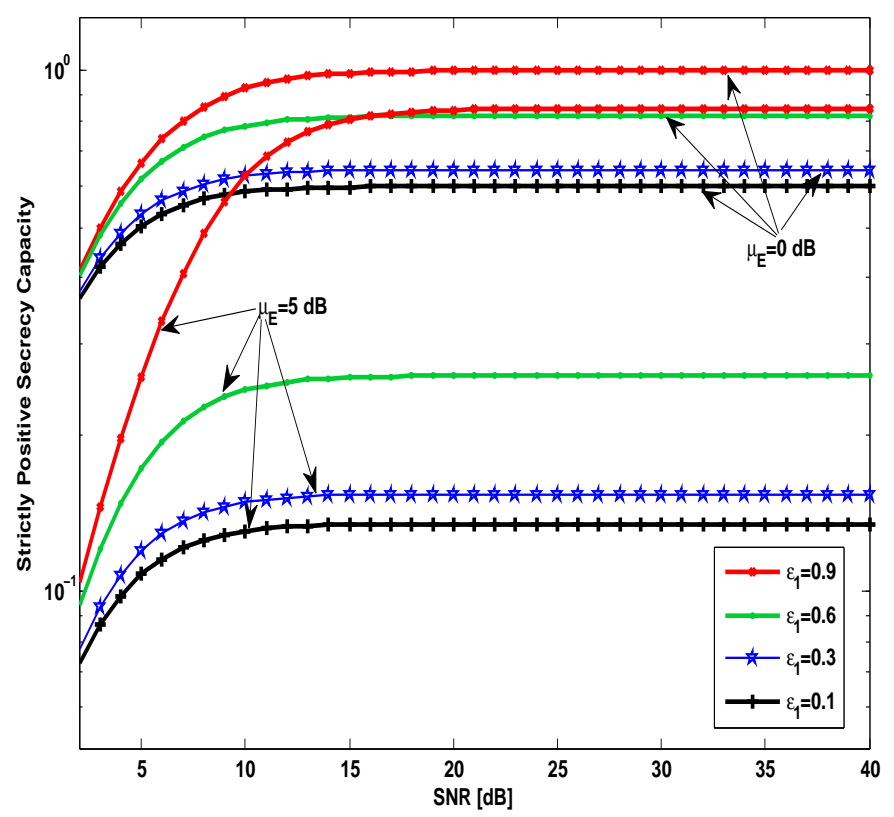

Fig. 3: Strictly Positive Secrecy Capacity for NOMA-AF relaying system for different values of $\epsilon_{1}$ and $\mu_{E}$ assuming that $m=2$ and $\alpha_{0}=\alpha_{2}=\alpha_{E}=1$

results, the more the values of SNR augment, the more the strictly positive secrecy capacity of NOMA-AF and NOMA-DF relaying networks goes to be a constant, which is demonstrated in section 4 . Therefore, we can conclude that the secrecy performance depends on several parameters such as $m, \mu_{E}$ and $\epsilon_{1}$.

\section{Conclusion}

In this paper, we have analyzed the secrecy performance of NOMA-AF relaying system and NOMA-DF relaying system in terms of closed-form exact and asymptotic expressions for strictly positive secrecy capacity under Nakagami$\mathrm{m}$ fading channels. We have shown that both systems have an identical secrecy performance for high SNR values. Furthermore, asymptotic results show that the strictly positive secrecy capacity for NOMA-AF and NOMA-DF relaying systems tends to be a constant for high values of SNR. We have also observed that various parameters can affect the strictly positive secrecy capacity such as $m, \mu_{E}$ and $\epsilon_{1}$. 


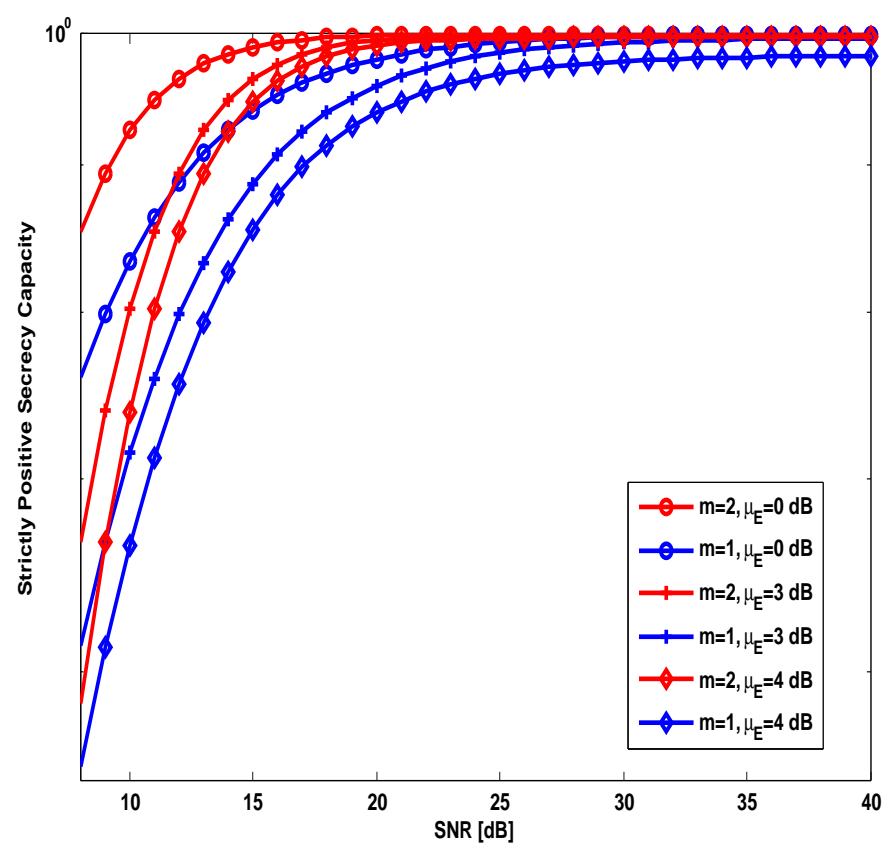

Fig. 4: Strictly Positive Secrecy Capacity for NOMA-AF relaying system when varying $\mu_{E}$ for different values of Nakagami-m fading parameter $(m=1,2)$ when $\epsilon_{1}=0.95$ and $\alpha_{0}=$ $\alpha_{2}=\alpha_{E}=1$

\section{References}

1. Y. Saito, Y. Kishiyama, A. Benjebbour, T. Nakamura, A. Li, and K. Higuchi, "Nonorthogonal multiple access (noma) for cellular future radio access," in 2013 IEEE 77th Vehicular Technology Conference (VTC Spring), 2013, pp. 1-5.

2. Z. Ding, Z. Yang, P. Fan, and H. V. Poor, "On the performance of non-orthogonal multiple access in $5 \mathrm{~g}$ systems with randomly deployed users," IEEE Signal Processing Letters, vol. 21, no. 12, pp. 1501-1505, 2014.

3. S. Timotheou and I. Krikidis, "Fairness for non-orthogonal multiple access in 5g systems," IEEE Signal Processing Letters, vol. 22, no. 10, pp. 1647-1651, 2015.

4. Y. Saito, A. Benjebbour, Y. Kishiyama, and T. Nakamura, "System-level performance evaluation of downlink non-orthogonal multiple access (noma)," in 2013 IEEE 24th Annual International Symposium on Personal, Indoor, and Mobile Radio Communications (PIMRC), 2013, pp. 611-615.

5. T. M. Cover and J. A. Thomas, Elements of Information Theory. USA: WileyInterscience, 1991.

6. Z. Ding, M. Peng, and H. V. Poor, "Cooperative non-orthogonal multiple access in $5 \mathrm{~g}$ systems," IEEE Communications Letters, vol. 19, no. 8, pp. 1462-1465, 2015. 


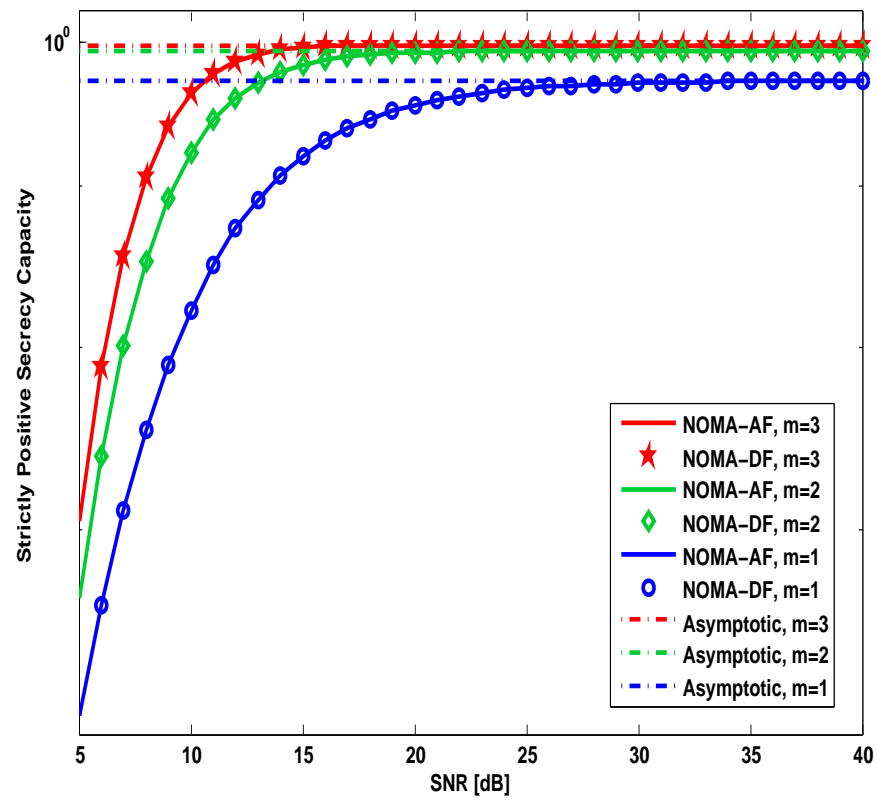

Fig. 5: Comparison between NOMA-AF relaying system and NOMA-DF relaying system for multiple values of Nakagami-m fading parameter $(m=1,2,3), \mu_{E}=0 d B, \epsilon_{1}=0.85$ and $\alpha_{0}=\alpha_{2}=\alpha_{E}=1$

7. Y. Liu, Z. Ding, M. Elkashlan, and H. V. Poor, "Cooperative non-orthogonal multiple access with simultaneous wireless information and power transfer," IEEE Journal on Selected Areas in Communications, vol. 34, no. 4, pp. 938-953, 2016.

8. N. Zaghdoud, W. H. Alouane, H. Boujemaa, A. B. Mnaouer, and F. Touati, "Secrecy performance of af relaying in cooperative noma over rician channel," in 2019 15th International Wireless Communications Mobile Computing Conference (IWCMC), 2019, pp. 805-810.

9. Y. Liu, G. Pan, H. Zhang, and M. Song, "Hybrid decode-forward amplify-forward relaying with non-orthogonal multiple access," IEEE Access, vol. 4, pp. 4912-4921, 2016.

10. X. Liang, Y. Wu, D. W. K. Ng, Y. Zuo, S. Jin, and H. Zhu, "Outage performance for cooperative noma transmission with an af relay," IEEE Communications Letters, vol. 21, no. 11, pp. 2428-2431, 2017.

11. Z. Ding, H. Dai, and H. V. Poor, "Relay selection for cooperative noma," IEEE Wireless Communications Letters, vol. 5, no. 4, pp. 416-419, 2016.

12. Y. Shiu, S. Y. Chang, H. Wu, S. C. . Huang, and H. Chen, "Physical layer security in wireless networks: a tutorial," IEEE Wireless Communications, vol. 18, no. 2, pp. 66-74, 2011.

13. Y. Zou, X. Wang, and W. Shen, "Optimal relay selection for physical-layer security in cooperative wireless networks," IEEE Journal on Selected Areas in Communications, vol. 31, no. 10, pp. 2099-2111, 2013. 
14. M. Zhang and Y. Liu, "Energy harvesting for physical-layer security in ofdma networks," IEEE Transactions on Information Forensics and Security, vol. 11, no. 1, pp. 154-162, 2016.

15. Y. Zou, X. Wang, and W. Shen, "Physical-layer security with multiuser scheduling in cognitive radio networks," IEEE Transactions on Communications, vol. 61, no. 12, pp. 5103-5113, 2013.

16. Z. Qin, Y. Liu, Z. Ding, Y. Gao, and M. Elkashlan, "Physical layer security for $5 \mathrm{~g}$ non-orthogonal multiple access in large-scale networks," in 2016 IEEE International Conference on Communications (ICC), 2016, pp. 1-6.

17. B. He, A. Liu, N. Yang, and V. K. N. Lau, "On the design of secure non-orthogonal multiple access systems," IEEE Journal on Selected Areas in Communications, vol. 35, no. 10, pp. 2196-2206, 2017.

18. J. Chen, L. Yang, and M. Alouini, "Physical layer security for cooperative noma systems," IEEE Transactions on Vehicular Technology, vol. 67, no. 5, pp. 4645-4649, 2018.

19. Z. Ding, L. Dai, and H. V. Poor, "Mimo-noma design for small packet transmission in the internet of things," IEEE Access, vol. 4, pp. 1393-1405, 2016.

20. C. S. Patel, G. L. Stuber, and T. G. Pratt, "Statistical properties of amplify and forward relay fading channels," IEEE Transactions on Vehicular Technology, vol. 55, no. 1, pp. 1-9, 2006.

21. Z. Ding, Y. Liu, J. Choi, Q. Sun, M. Elkashlan, I. Chih-Lin, and H. V. Poor, "Application of non-orthogonal multiple access in lte and $5 \mathrm{~g}$ networks," IEEE Communications Magazine, vol. 55, no. 2, pp. 185-191, 2017.

22. Y. Liu, Z. Qin, M. Elkashlan, Y. Gao, and L. Hanzo, "Enhancing the physical layer security of non-orthogonal multiple access in large-scale networks," IEEE Transactions on Wireless Communications, vol. 16, no. 3, pp. 1656-1672, 2017. 
Figures

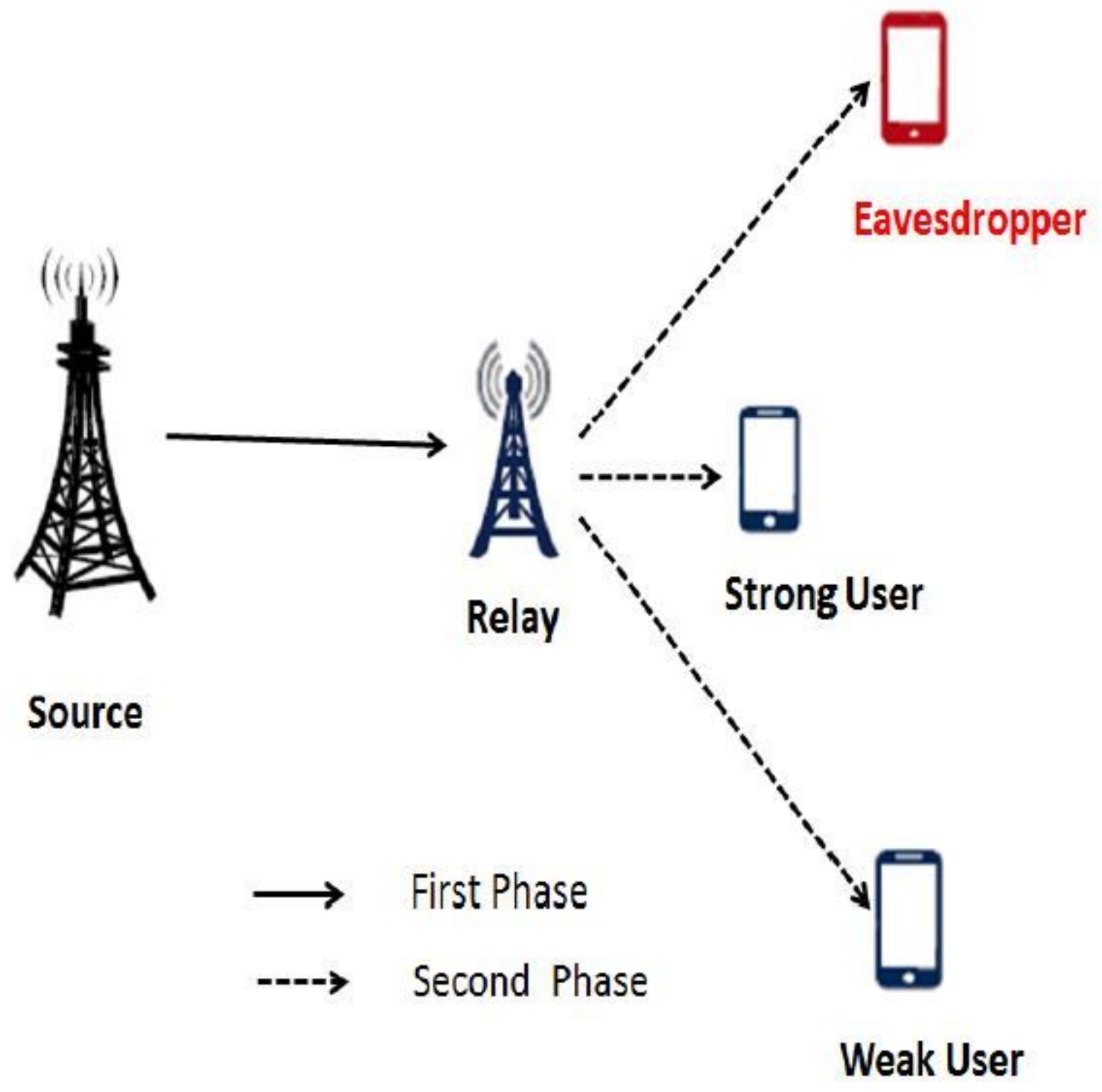

Figure 1

System architecture 


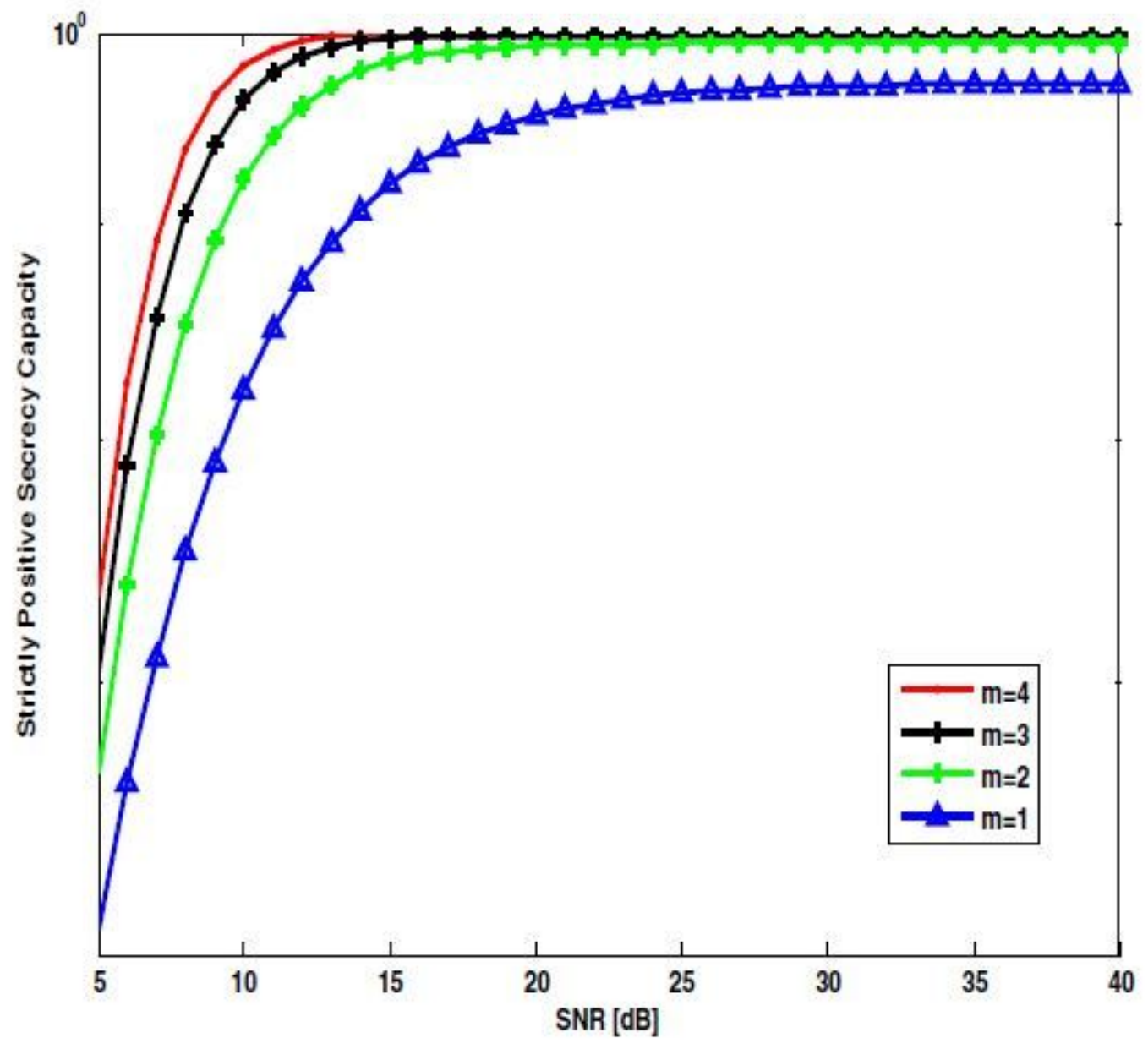

Figure 2

"Please see the Manuscript PDF file for the complete figure caption". 


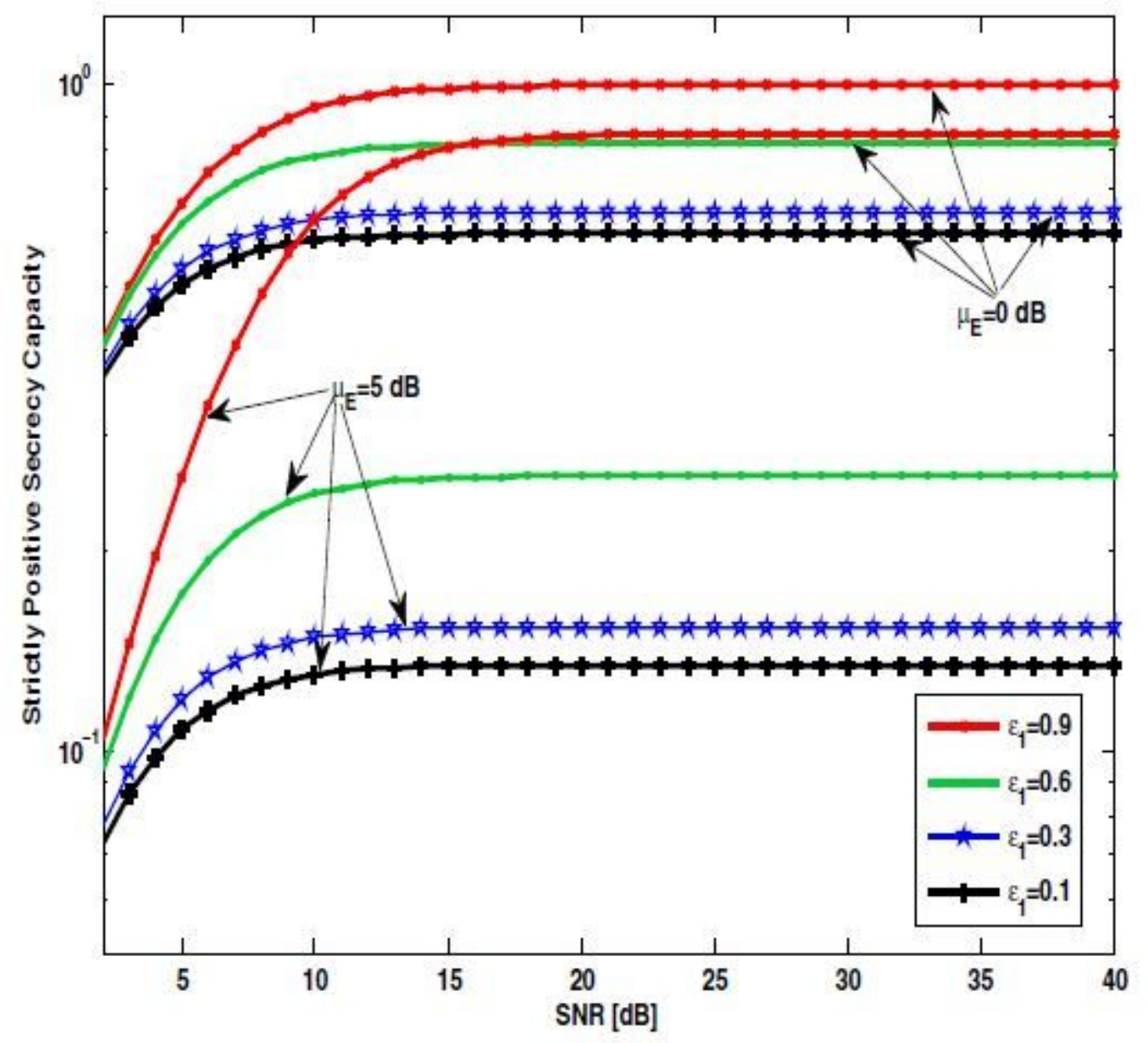

Figure 3

"Please see the Manuscript PDF file for the complete figure caption". 


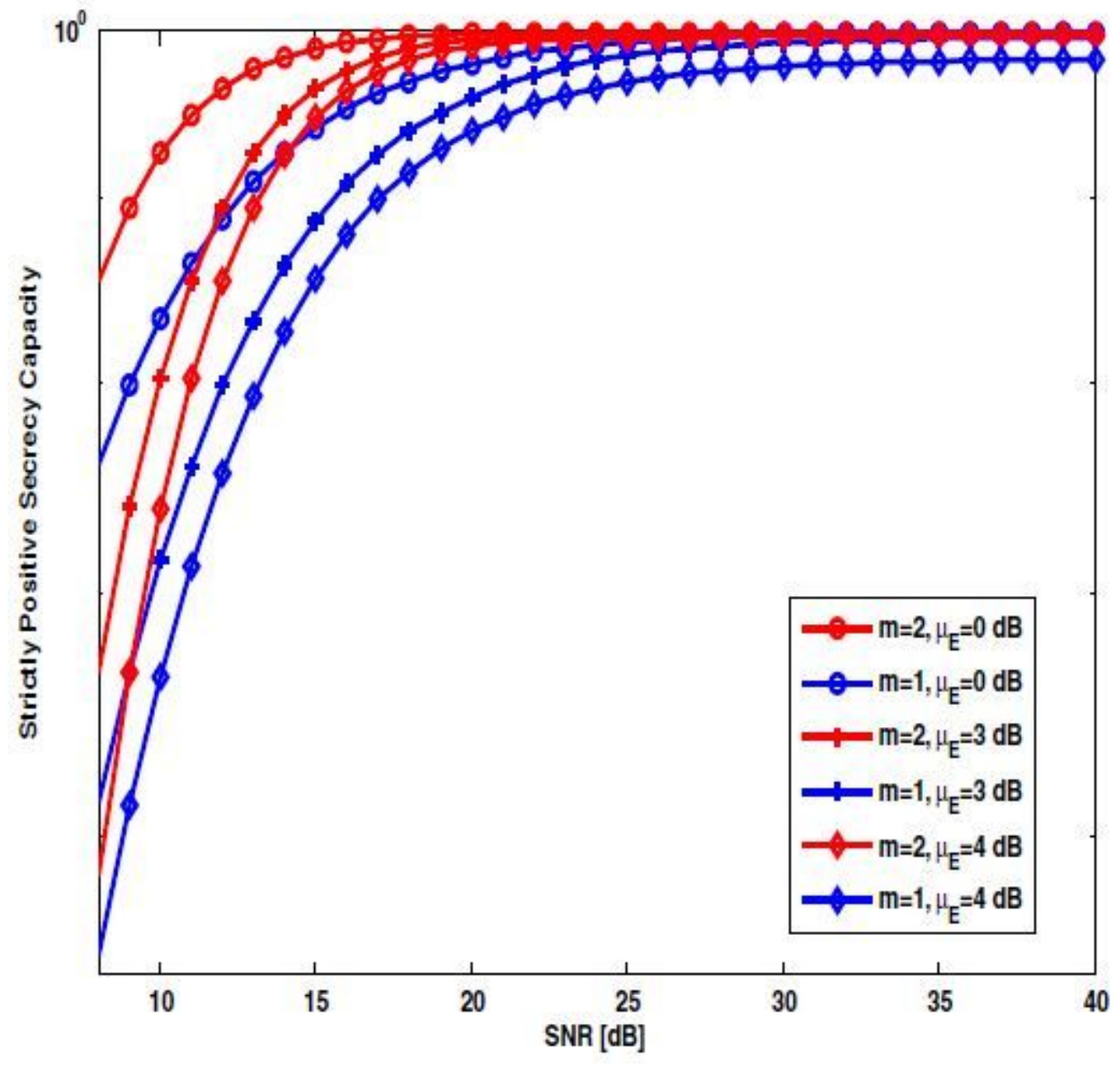

Figure 4

"Please see the Manuscript PDF file for the complete figure caption". 


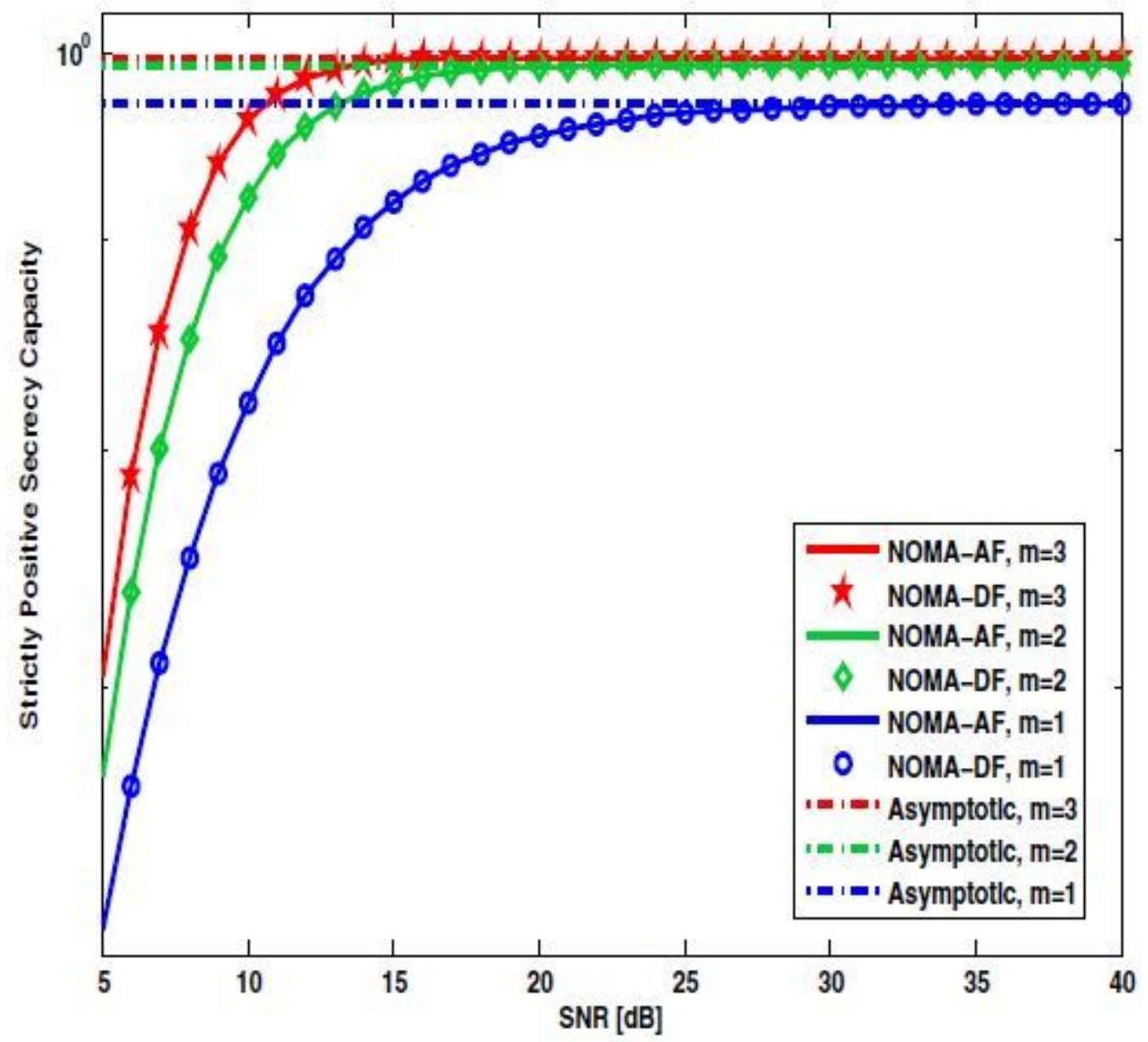

Figure 5

"Please see the Manuscript PDF file for the complete figure caption". 